\title{
Eficacia y seguridad de la vacuna contra el dengue
}

\author{
Efficacy and safety of dengue vaccine
}

María Florencia Herrera Bornes ${ }^{a}$

\begin{abstract}
Resumen
El dengue es un problema creciente para la salud pública mundial. En Argentina, los casos se han ido incrementado en los últimos años. La vacuna Dengvaxia (CYD-TDV) fue aprobada por la Agencia Nacional de Medicamentos, Alimentos y Tecnología (ANMAT) en 2017, y actualmente está indicada para personas entre los 9 y 45 años de edad que residan en zonas endémicas.

A partir de la consulta de una paciente sobre la posibilidad de vacunarse contra el dengue, la autora se plantea la pertinencia de su indicación, teniendo en cuenta la eficacia y seguridad de la vacuna. Luego de una búsqueda rápida se encontró evidencia que señala que la vacuna contra el dengue CYD-TDV mostró poca eficacia en comparación con otras vacunas disponibles en el mercado, siendo más segura y eficaz en personas que ya han sido infectadas anteriormente por el virus del dengue (sujetos seropositivos). En cambio, se observó un aumento del riesgo de dengue grave en los infectados por vez primera tras la vacunación (sujetos seronegativos). Se concluye que la estrategia recomendada consiste en vacunar únicamente a las personas que hayan tenido infección por dengue con anterioridad, consistiendo en una buena práctica la toma de decisiones compartidas con cada paciente.
\end{abstract}

\begin{abstract}
Dengue is a growing problem for global public health. In Argentina, cases have been increasing in recent years. The Dengvaxia vaccine (CYD-TDV) was approved by the National Agency for Medicines, Food and Technology in 2017, and it is currently indicated for people between 9 and 45 years of age who reside in endemic areas.

Based on the consultation of a patient about the possibility of being vaccinated against dengue, the author considers the relevance of its indication, taking into account the efficacy and safety of the vaccine. After a quick search, evidence was found that indicates that the CYD-TDV dengue vaccine showed little efficacy compared to other vaccines available on the market, being safer and more effective in people who have already been previously infected by the dengue virus (seropositive subjects). In contrast, an increased risk of severe dengue was observed in those infected for the first time after vaccination (seronegative subjects). It is concluded that the recommended strategy consists of vaccinating only people who have had dengue infection before, making shared decisions with each patient a good practice.
\end{abstract}

Palabras clave: Dengue, Vacunas contra el Dengue, Medicina Basada en la Evidencia. Keywords: Dengue, Dengue Vaccines, Evidence-Based Medicine.

Herrera Bornes, MF. Eficacia y seguridad de la vacuna contra el dengue. Evid Actual Pract Ambul. 2021;24(1):e002064.

\section{Escenario clínico}

Se presenta en el consultorio del médico de familia una mujer de 35 años de edad, sin antecedentes patológicos relevantes, y manifiesta sentirse preocupada porque un familiar tuvo dengue. Ella relata que hay una vacuna contra el dengue en un centro de vacunación privado, y consulta si sería conveniente su aplicación, preguntando si es efectiva para prevenir la enfermedad y si le podría provocar algún daño, teniendo además en cuenta su alto costo.

\section{Pregunta que generó el caso}

En población general, la vacuna contra el dengue ¿es efectiva para prevenir la infección y/o disminuir la gravedad de la enfermedad causada por este agente? ¿Es segura su aplicación en pacientes sanos?

\section{Algunos datos sobre el dengue}

El dengue es una enfermedad febril causada por una infección viral transmitida a través de la picadura de un mosquito perteneciente al género Aedes. El espectro de manifestaciones de la infección por dengue es amplio, puede cursar de manera asintomática o producir cuadros clínicos de diversa gravedad ${ }^{1,2}$.

Luego de un periodo de incubación variable, entre tres y 14 días tras la picadura de un mosquito infectado, los síntomas más frecuentes en las formas clínicamente aparentes incluyen la presencia de fiebre, malestar general, cefalea, dolor retro-ocular, mialgias y artralgias ${ }^{2}$. Alrededor de la mitad de los casos presentan además manifestaciones cutáneas, que consisten en un exantema macular o maculopapular que puede ser pruriginoso, se distribuye en forma variable en el rostro, el tórax, el abdomen o los miembros, y suele aparecer dos a cinco días luego del comienzo de la fiebre ${ }^{1}$.

En los casos con evolución a cuadros graves, a partir de los tres a siete días del transcurso de la enfermedad, se pueden observar manifestaciones hemorrágicas (habitualmente petequias o equimosis, o con menos frecuencia, hematemesis, menorragia, melena, epistaxis), y extravasación de plasma debido al aumento de la permeabilidad vascular, con aumento del hematocrito y colecciones líquidas en cavidades serosas (derrame pleural, ascitis derrame pericárdico). En los casos más severos, puede presentarse cuadros de shock, distrés respiratorio, sangrados graves y falla orgánica ${ }^{1,2}$. La evolución en etapas de la enfermedad por dengue (etapa febril, crítica y de recuperación o convalescencia) permite el seguimiento de los pacientes para detectar señales de alarma (ver Tabla 1 y figura 1), para proporcionar el tratamiento de sostén necesario, prevenir el shock y disminuir la mortalidad por este problema de salud, ya que no existe una terapia antiviral directa disponible ${ }^{2}$

\footnotetext{
a Sericio de medicina familiar y comunitaria, Hospital Italiano de Buenso Aires. mariaflorenciaherrerabornes@gmail.com
} 
Tabla 1. Banderas rojas o signos de alarma que preceden el shock en pacientes con dengue.

\section{Signos y síntomas}

Dolor abdominal intenso y sostenido

Vómitos persistentes

Derrame seroso (en peritoneo, pleura o pericardio) detectado por clínica, por laboratorio (hipoalbuminemia) o por imágenes (ecografía de abdomen o radiografía de tórax)

\section{Sangrado de mucosas}

Cambio en el estado mental del paciente: somnolencia o irritabilidad sobre todo en niños.

Incremento brusco del hematocrito concomitante con rápida disminución del recuento de plaquetas

Fuente: Dengue. Guía para el equipo de Salud. Ministerio de Salud de la Nación Argentina, 2016.

El virus del dengue pertenece a la familia Flaviviridae y existen cuatro variantes: DEN-1, DEN-2, DEN-3 y DEN-4. Cualquier serotipo puede producir formas graves de la enfermedad, aunque los serotipos 2 y 3 han sido asociados a la mayor cantidad de casos graves y fallecidos ${ }^{2}$.

La inmunidad conferida por la infección por el virus dengue es serotipo-específica. Esto significa que la infección con un serotipo determinado confiere inmunidad permanente contra el mismo (inmunidad homóloga), mientras que sólo por unos meses se observa protección cruzada contra la enfermedad causada por los otros tres serotipos de dengue (inmunidad heteróloga, de corta duración $)^{2,3}$

Por otro lado se ha documentado que los casos de dengue grave son más frecuentes en las personas que ya padecieron dengue por un serotipo (infección primaria) y luego adquieren la infección por un serotipo diferente al anterior (infección secundaria heterotípica) ${ }^{2}$.

Figura 1. Etapas de evolución del dengue. Fuente: Dengue. Guía para el equipo de Salud. Ministerio de Salud de la Nación, Argentina, 2016.

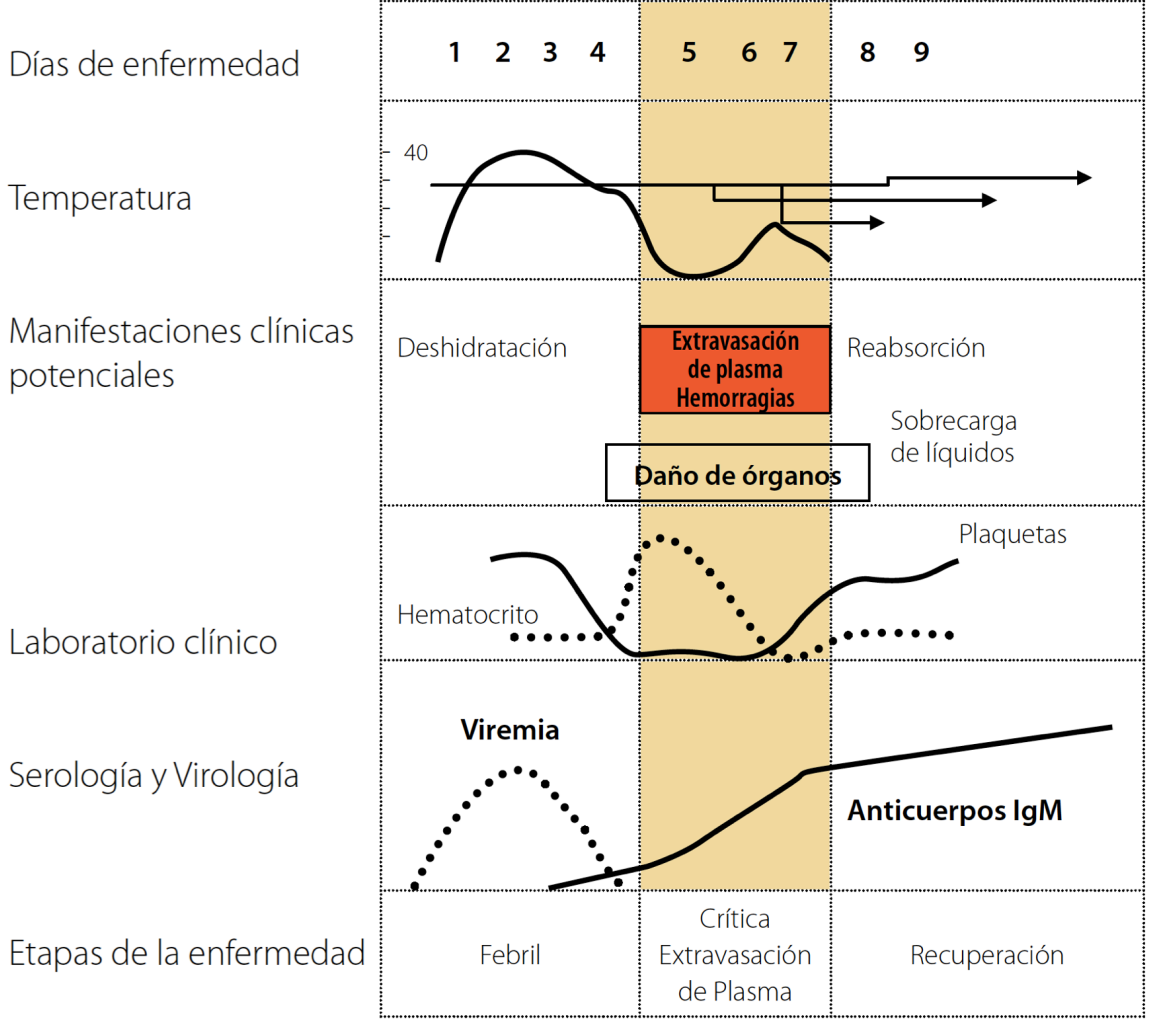

El Aedes aegypti es el vector más frecuente de la enfermedad en América. Este mosquito tiene hábitos domiciliarios, por lo que la transmisión es predominantemente doméstica. Las medidas de prevención centradas en el control de los mosquitos son eficaces, pero difíciles de sostener en el tiempo y requieren muchos recursos. Algunas estrategias incluyen la reducción de los sitios de reproducción (mediante la educación comunitaria para disminuir los potenciales recipientes que puedan acumular agua), el control de las larvas, y el uso de insecticidas ${ }^{4}$. Las medidas de protec- ción personales, para prevenir las picaduras del vector, incluyen el uso de ropa protectora y la aplicación de repelentes químicos ${ }^{5}$.

Dado que la infección por un serotipo de dengue proporciona protección a largo plazo contra la re-infección con ese mismo serotipo, el desarrollo de una vacuna eficaz contra el dengue podría constituir una herramienta valiosa para el control de esta enfermedad, que constituye un problema de salud pública creciente en las últimas décadas, aumentando su incidencia, expandiéndose geográficamente a diferentes países y afectando tanto 
a áreas urbanas como rurales ${ }^{6}$.

La primera vacuna contra el dengue es una vacuna contra el virus del dengue vivo quimérico, recombinante y tetravalente llamada CYD-TDV. Fue desarrollada por Sanofi Pasteur y comercializada bajo el nombre Dengvaxia ${ }^{\circledR}$. En 2016, Brasil fue uno de los primeros países en adoptarla ${ }^{7}$. En Argentina, su comercialización fue aprobada por ANMAT en $2017^{8}$, y actualmente ha sido autorizada en 20 países con indicación para personas entre los 9 y 45 años de edad que residan en zonas endémicas ${ }^{9}$.

Además existen otras vacunas que se encuentran en evaluación y que aún no han sido autorizadas por las agencias regulatorias.

\section{Estrategia de búsqueda}

Se realizó una búsqueda en PubMed utilizando como palabras claves "vaccination", "dengue" y effectiveness". Limitando las publicaciones a los últimos cinco años y aplicando los filtros para ensayos clínicos aleatorizados y revisiones sistemáticas, se recuperaron 37 artículos.

\section{Resumen de la evidencia}

Se seleccionaron dos revisiones sistemáticas ${ }^{10,11}$ que parecían responder a la pregunta que motivó este trabajo.

Da Silveira LTC, et al. Systematic review of dengue vaccine efficacy. BMC Infect Dis. 2019;19(1):750.

Objetivo. Estimar la eficacia de la vacuna contra el dengue disponible comercialmente en Brasil (Dengvaxia ${ }^{\circledR}$ ) para reducir los casos sintomáticos de dengue en la población general.

Diseño. Revisión sistemática y meta-análisis.

Estrategia de búsqueda. Se incluyeron ensayos clínicos controlados aleatorizados (ECCA) publicados entre 2000 y 2017 , identificados en las bases de datos MEDLINE(Pubmed), LILACS, Cochrane Library, y EMBASE. La selección fue realizada por dos revisores independientes, recurriendo a un tercer revisor en caso de desacuerdos.

Resultados. Se incluyeron siete ensayos clínicos, con un total de 36.371 participantes, con edades comprendidas entre 2 y 45 años. El meta-análisis con el modelo de efectos aleatorios, estimó la eficacia de la vacuna en $44 \%$, con un rango de 25 a $59 \%$ y alta heterogeneidad $\left(I^{2}=80,1 \%\right)$. Luego de evaluar el efecto de la seropositividad, el serotipo y la edad mediante meta-regresión, no se encontró una explicación para la heterogeneidad observada. Sin embargo, el análisis estratificado mostró baja heterogeneidad para el serotipo 4 (10,3\%) y significativa para el serotipo 2 $(64,5 \%)$. Por este motivo, se interpreta que el efecto de la vacuna podría no ser uniforme para los distintos serotipos del virus, y este efecto podría ser responsable de la alta heterogeneidad encontrada en el meta-análisis. La mayoría de las personas vacunadas tenían inmunidad previa para al menos un serotipo, lo que generó preocupaciones por la seguridad en personas sin inmunidad previa.

Conclusiones. La vacuna contra el dengue mostró poca eficacia contra el dengue sintomático, especialmente contra los serotipos 1 y 2. Los autores anticipan que el impacto del uso de la vacuna CYD-TDV como estrategia de prevención primaria será limitado.

Rodrigues Rosa B, et al. Efficacy, immunogenicity and safety of a recombinant tetravalent dengue vaccine (CYD-TDV) in children aged 2-17 years: systematic review and meta-analysis. BMJ Open. 2019;9(3):e019368

Objetivo. Evaluar la eficacia, inmunogenicidad y seguridad de la vacuna CYD-TDV en la prevención del dengue en niños de 2 a 17 años.

Diseño. Revisión sistemática y meta-análisis.
Fuente de datos. MEDLINE, EMBASE y Cochrane (hasta el 5 de diciembre de 2018).

Criterios de elegilibilidad de los estudios. ECCA que compararon la eficacia, inmunogenicidad y seguridad de la vacuna CYD-TDV contra placebo $u$ otras vacunas para prevenir casos de dengue en niños de 2 a 17 años de edad.

Resultados principales. Se incluyeron nueve estudios con un total de 34.248 participantes. La eficacia global de la vacuna CYD-TDV fue del $60 \%$ (Riesgo Relativo [RR] 0,40; Intervalo de Confianza [IC] del $95 \% 0,30$ a 0,54). La eficacia serotipoespecífica de la vacuna fue de $51 \%$ para DEN-1 (RR 0,49; IC95\% 0,39 a 0,63 ); $34 \%$ para DEN-2 (RR 0,66; IC95\% 0,50 a 0,86); $75 \%$ para DEN-3 (RR 0,25; 0,18 a 0,35) y $77 \%$ para DEN-4 (RR 0,23; IC95\% 0,15 a 0,34). Los eventos adversos más comunes fueron la cefalea y el dolor en el sitio de inyección.

Limitaciones. La principal limitación de este estudio fueron la disponibilidad de datos incompletos o no del todo claros.

Conclusiones e implicancias de los hallazgos clave. La vacuna CYD-TDV se considera segura y capaz de proteger parcialmente a los niños y adolescentes contra los cuatro serotipos del virus dengue por un periodo de un año. Sin embargo, se deberían priorizar las investigaciones para mejorar la eficacia de la vacuna, para proveer mayor protección a largo plazo frente a todos los serotipos virales.

\section{Conclusiones y recomendaciones}

Los casos de dengue en Argentina se han ido incrementando en los últimos años. Durante el 2016, en la Argentina se notificaron más de 41.000 casos, un número sin precedentes con predominio del serotipo 1 (más del $98 \%)^{12}$. Para la temporada 2019-2020, se notificaron al Sistema Nacional de Vigilancia de la Salud 96.454 casos con sospecha de dengue. Se registraron 56.293 casos de dengue (entre probables y confirmados por laboratorio o nexo epidemiológico) sin antecedente de viaje, 1.498 con antecedente de viaje al exterior $u$ a otras provincias y otros 1.098 cuyo lugar de adquisición se encuentra aún en investigación. En esta temporada se registró la presencia de 3 serotipos. De los casos serotipificados el $72 \%$ correspondió a DEN-1, 26\% a DEN-4 y $2 \%$ DEN- $2^{13}$. El origen de este aumento se relaciona con varios factores: el cambio climático, la adaptación del mosquito para resistir menores temperaturas, la falta de control de los vectores, la globalización, entre otras causas.

La Organización Mundial de la Salud (OMS) recomienda que los países consideren la posibilidad de introducir la vacuna CYD TDV contra el dengue solo en entornos geográficos en los que los datos epidemiológicos indiquen que hay una gran carga de enfermedad (seroprevalencia del $70 \%$ o más). Se desaconseja su implementación en poblaciones con seroprevalencia menor al $50 \%$, debido a que su eficacia será escasa y aumentará el riesgo, a largo plazo, de que los individuos seronegativos desarrollen dengue grave con una infección posterior ${ }^{14}$. El esquema de vacunación consiste en la aplicacion de tres dosis, administradas a intervalos de 6 meses, a partir de los 9 años de edad, y sus contraindicaciones se enumeran en la Tabla $2^{14}$. 
Tabla 2. Contraindicaciones de la vacuna CYD-TDV (Dengvaxia $\left.{ }^{\circledR}\right)$ informadas por el fabricante. Fuente: OMS ${ }^{14}$

\section{Situaciones que contraindican la aplicación de la vacuna contra el} dengue a virus vivos atenuados

Antecedentes de reacciones alérgicas graves a cualquier componente de la vacuna o a la administración previa de la vacuna contra el dengue $u$ otra vacuna que contenga los mismos componentes

Inmunodeficiencia congénita o adquirida, con compromiso de la inmunidad celular

Infección sintomática por el VIH o asintomática por este virus acompañada de signos de inmunodeficiencia

Embarazo

Lactancia

Estas recomendaciones son consistentes con los resultados de los metanálisis resumidos en este artículo ${ }^{10,11}$, en los que se evidencia que la vacuna disponible comercialmente (Dengvaxia ${ }^{\circledR}$ ) mostró poca eficacia comparada contra placebo $u$ otras vacunas utilizadas en la actualidad, y además toma en cuenta los informes que indican la posibilidad de un cuadro clínico más severo del dengue, particularmente en individuos vacunados que no tienen una respuesta inmune al DENV-2 pero que producen anticuerpos contra este serotipo. Un análisis retrospectivo de los datos obtenidos en tres ensayos clínicos patrocinados por Sanofi reveló que, entre los participantes seronegativos al momento de la vacunación, las incidencias acumuladas de hospitalizaciones por dengue y de dengue grave a los 5 años de seguimiento fue mayor en quienes recibieron la vacuna con respecto a quienes se encontraban en el grupo control, mientras el riesgo de presentar estos desenlaces fue menor en los participantes seropositivos al momento de recibir la vacuna que en los controles seropositivos. Se concluyó que la vacuna CYD-TDV confiere protección frente al dengue severo y a las hospitalizaciones por dengue en las personas que habían tenido exposición al patógeno antes de la inmunización, y se constató un mayor riesgo de estos desenlaces en las personas vacunadas sin exposición previa ${ }^{15}$. Esta información fundamenta la estrategia recomendada para los países que estén pensando en incluir la vacunación en sus programas de control del dengue, que consiste en determinar el estado serológico de los sujetos antes de la vacunación y en vacunar únicamente a los que ya hayan sido infectados con anterioridad, de acuerdo con los resultados de una prueba de anticuerpos o con la demostración de una infección previa confirmada mediante pruebas de laboratorio ${ }^{9}$.

Por todo lo expuesto anteriormente, y teniendo en cuenta la evidencia hasta la fecha en cuanto a la eficacia y seguridad de la vacuna contra el dengue que se encuentra disponible comercialmente en Argentina y otros países, considero que sería una buena práctica la toma de decisiones compartidas con el paciente, haciendo hincapié en la importancia de la continuidad de las medidas de control de vectores.

Recibido el 20/06/2020 Aceptado el 18/12/2020 y Publicado el 03/02/2021.

\section{Referencias}

1. Thomas SJ, Rothman AL, Srikiatkhachorn A, Kalayanarooj S, et al. Dengue virus infection: Clinical manifestations and diagnosis. In: Post TW, et al., editors. UpToDate. Waltham MA: UpToDate Inc; 2019.Available from: https://www.uptodate.com/contents/dengue-virus-infection-clinicalmanifestations-and-diagnosis.

2. Enfermedades infecciosas "Dengue", Guía para el equipo de salud. Ministerio de Salud de la Nación Argentina; 2015. Available from: https: //bancos.salud.gob.ar/sites/default/files/2018-10/0000000062cnt-guia-dengue-2016.pdf.

3. Endy TP, Nisalak A, et al. Relationship of preexisting dengue virus (DV) neutralizing antibody levels to viremia and severity of disease in a prospective cohort study of DV infection in Thailand. 2004;Available from: 10.1086/382280;https://pubmed.ncbi.nlm.nih.gov/14999601/.

4. Thomas SJ, Rothman AL, Srikiatkhachorn A, Kalayanarooj S, et al. Dengue virus infection: Prevention and treatment. In: Post TW, et al., editors. UpToDate. Waltham MA: UpToDate Inc; 2019.Available from: https://www.uptodate.com/contents/dengue-virus-infection-prevention-and-treatment.

5. Breisch NL. Prevention of arthropod and insect bites: Repellents and other measures. In: Post TW, et al., editors. UpToDate. Waltham MA: UpToDate Inc; 2020.Available from: https://www.uptodate.com/contents/prevention-of-arthropod-and-insect-bites-repellents-and-other-measures.

6. Informe ultrarrápido de evaluación de tecnología sanitaria. Eficacia y seguridad de la vacuna tetravalente para Dengue (DengvaxiaNR). Buenos Aires: Administración Nacional de Medicamentos, Alimentos y Tecnología (ANMAT); 2017. Available from: http://www.anmat.gov.ar/ets/ETS_DENGUE_ agosto-EN2.pdf.

7. Bases técnicas e científicas da conclusão da análise do registro do medicamento aprovado. Vacina dengue 1, 2, 3 e 4 (recombinante, atenuada), Dengvaxia $^{\circledR}$. Brasilia, Brasil: Agência Nacional de Vigilância Sanitária - Anvisa; 2016. Available from: https://www.pharmabiz.net/wp-content/uploads/ 2016/03/registrodengvaxia.pdf.

8. Disposición Número 3135. Expediente número 1-47-10281-15-4. Buenos Aires: Administración Nacional de Medicamentos, Alimentos y Tecnología (ANMAT); 2017. Available from: http://www.anmat.gov.ar/boletin_anmat/marzo_2017/Dispo_3135-17.pdf.

9. WHO. Dengue vaccine: WHO position paper, September 2018 - Recommendations. Vaccine. 2019;37(35):4848-4849. Available from: 10.1016/j. vaccine.2018.09.063.

10. da Silveira LC, Tura B, Santos M. Systematic review of dengue vaccine efficacy. BMC Infect Dis. 2019;19(1):750. Available from: 10.1186/s12879019-4369-5; https://pubmed.ncbi.nlm.nih.gov/31455279/.

11. Rodrigues-Rosa $B$, da Cunha AJLA, de Andrade-Medronho R. Efficacy, immunogenicity and safety of a recombinant tetravalent dengue vaccine (CYD-TDV) in children aged 2-17 years: systematic review and meta-analysis. BMJ Open. 2019;9(3):e019368. Available from: 10.1136/bmjopen2017-019368.

12. Argentina. Ministerio de Salud de la Nación. Boletín Integrado de Vigilancia № 340 - SE 51. Diciembre de 2016; 2016 . Available from: http: //www.msal.gob.ar/images/stories/boletines/boletin_integrado_vigilancia_N340-SE51.pdf.

13. Agentina. Ministerio de Salud de la Nación. Boletin integrado de vigilancia $N^{\circ} 507$ SE 31/2020; 2020. Available from: https://www.argentina.gob.ar/ sites/default/files/biv 507 se 31.pdf.

14. Organización Mundial de la Salud. Vacuna contra el dengue: documento de posición de la OMS, julio de 2016. Weekly Epidemiological Record. 2016;91(30):349-364. Available from: https://www.who.int/immunization/policy/position_papers/who_pp_dengue_july2016_ES.pdf?ua=1.

15. Sridhar S, Luedtke A, Langevin E, et al. Effect of Dengue Serostatus on Dengue Vaccine Safety and Efficacy. N Engl J Med. 2018;379(4):327-340. Available from: 10.1056/NEJMoa1800820. 